\title{
L'USO DELLE SUBORDINATE GERUNDIALI NELLA SCRITTURA SCOLASTICA E UNIVERSITARIA
}

\author{
Michela Dota ${ }^{1}$
}

\section{INTRODUZIONE}

Il presente lavoro indaga l'impiego del gerundio subordinativo in alcune scritture scolastiche (prodotte in istituti superiori di secondo grado) e universitarie (di triennalisti iscritti a corsi umanistici), al fine di rilevare la vitalità attuale e le criticità più frequenti nell'uso di questa struttura nella popolazione ancora coinvolta in un ciclo d'istruzione. Quest'ultima circostanza permette ovviamente di utilizzare tali produzioni scritte come termometro della vitalità di quella struttura nell'attuale italiano scolastico - nell'ultimo decennio ritenuto baluardo dell'italiano standard (Antonelli, 2011), almeno nelle sue declinazioni manualistiche - e nella sua didattica; al contempo, proprio il carattere acerbo, o in assestamento, della competenza linguistica degli scriventi, per quanto maturata (anche) in un ambiente di apprendimento sorvegliato quale è la scuola o i laboratori di lingua e scrittura italiana in università, è interessante in quanto potenzialmente foriero dei nuovi usi e assetti che potrebbe assumere l'italiano neostandard.

Gli ultimi studi che documentano un'evoluzione del sistema neostandard per l'uso del gerundio subordinativo risalgono agli anni Novanta; in particolare Solarino (1991 e 1992), oltre a confermare lo stato di buona salute del gerundio semplice, in progressiva espansione nell'uso comune (Policarpi, 1974; Voghera, 1985) grazie all'ampliamento delle sue funzioni, rilevava l'affermarsi dell'uso sintatticamente iconico del gerundio presente, funzionale in primis all'espressione dell'anteriorità, nonché di altri rapporti logici (cfr. anche Serianni, 2006/2017: 171-172). Il favore per la giacitura iconica sarebbe più spiccato per i parlanti/scriventi inesperti o in formazione (quali sono gli adolescenti): considerando valida l'ipotesi della naturalezza ${ }^{2}$ (Mayerthaler, 1981; Wurzel, 1989; Dressler, Mayerthaler, Panagl, Wurzel, 1987), il costrutto iconico è di più facile accesso in parlanti/scriventi con interlingue non avanzate e quindi è presumibilmente più frequente nelle loro produzioni (Solarino, 1991 e 1992). L'ipotesi trovava conferma nell'analisi di un corpus costituito da «temi in classe prodotti nell'arco del biennio da 11 allievi di un istituto tecnico di Bari negli anni 1982-83» (Solarino, 1992: 17).

1 Università degli Studi di Milano.

2 Il principio di naturalezza è il cardine della Morfologia Naturale (MN), approccio teorico che spiega il mutamento linguistico come naturale tendenza della lingua a ridurre la propria marcatezza, al fine di ridurre la propria complessità e ottimizzare le potenzialità comunicative di ogni struttura. 
Per converso, lo studio documentava la significativa regressione del gerundio composto, che comunque resisteva, e resiste tuttora, nei testi scientifici, legislativi e burocratici ${ }^{3}$ (Dardano, 1994).

La duttilità di questo modo verbale, e l'ammissibilità di questi costrutti emergenti a dispetto dei vincoli posti dallo standard (su cui par. 2), sono inoltre documentabili nelle varietà di italiano regionale: osservando l'italiano regionale sardo contemporaneo, Caddeo (2019) ha rilevato la possibilità di ricorrere al gerundio per esprimere una predicazione riferita all'argomento oggetto dei verbi di percezione e in sostituzione di una proposizione pseudo-relativa introdotta dal cosiddetto "c'è presentativo" (es.: c'era gente aspettando da più di mezz'ora). Rispetto alla possibilità di accordo del gerundio con entrambi gli argomenti diretti di un verbo di percezione, è emerso come la disambiguazione della funzione circostanziale o predicativa - sia generalmente garantita dalla sua precisa posizione sintattica, di fatto vincolata.

Per quanto concerne, invece, le scritture scolastiche, il recente bilancio a cura di Ruele e Zuin (2020), effettuato su un corpus di tremila compiti scritti all'Esame di Stato del secondo ciclo di istruzione che coprono l'arco di tempo dal 2001 al 2016, conferma l'espansione del gerundio in funzione testuale, già segnalata in D'Achille (2010) a proposito dei tratti evolutivi dell'italiano contemporaneo; in aggiunta, il rapporto di ricerca documenta «la quasi grammaticalizzazione di espressioni come concludendo» (Ruele, 2020: 154).

L'eventuale persistenza e la consistenza della fenomenologia fin qui esposta sarà verificata nelle scritture menzionate in apertura, insieme all'eventuale insorgere di altri fenomeni. Si presterà poi attenzione alle già citate criticità morfosintattiche: per quanto non esclusive delle scritture giovanili (ed anzi documentate persino in scriventi esperti: Serianni, 2006/2017: 168 e ss.), esse segnalano dei punti di rottura nelle interlingue degli scriventi, che possono fornire elementi utili per una eventuale ricalibrazione didattica. Prima di presentare il corpus (par. 3) e illustrare l'analisi (par. 4 e ss.), si ritiene opportuno fornire una breve ricognizione preliminare sulla norma del gerundio subordinativo, trasmessa dalle grammatiche e dalla pratica didattica; la panoramica sarà utile per inquadrare al meglio i risultati dell'indagine.

\section{IL GERUNDIO TRA NORMA GRAMMATICALE E NORMA SCOLASTICA}

Alcuni studi (Antonini, 1974; Skytte, 1991; Barattella, 2015-2016: 8-10) hanno mostrato come nella tradizione descrittiva della grammatica italiana la trattazione del gerundio sia frammentaria ed eterogenea. In particolare, Skytte (1991), attraverso un ampio excursus nella grammaticografia italiana tra Quattrocento e Cinquecento, documenta l'egemonia di una descrizione del gerundio incentrata sull'ortografia, sulla morfologia e sulla stilistica, quale conseguenza del primato, conferito dai grammatici, alla trattazione morfologica e delle parti del discorso, sulla scorta del modello descrittivo latino. In questa tradizione, che trascura di osservare e illustrare le possibilità d'uso e i vincoli sintattici propri del gerundio, la grammatica del Buonmattei rappresenterebbe un punto di svolta: per la prima volta il gerundio è trattato in un capitolo autonomo, è considerato da un punto di vista

3 Per questi ultimi, in tempi più recenti, l'uso del gerundio sarebbe stato disincentivato per garantire una maggiore trasparenza del dettato (Cortelazzo, 2002); nella pratica, però, l'auspicio ha avuto finora scarso seguito (Cortelazzo, 2014b, 2015a e 2015b; Lubello, 2015: 271; Lubello, 2018). 
semantico-pragmatico e non solo morfologico. L'approccio tradizionale è comunque prevalente ed è replicato sino alle note e diffuse grammatiche postunitarie scolastiche, quali le fortunate Morandi, Cappuccini (1895: 199-200) e Petrocchi (1887: passim). Specificazioni di natura sintattica, non scevre da addentellati pragmatici, occorrono invece nella Sintassi italiana dell'uso moderno di Fornaciari (1884: 217-221) - che si sofferma sul costrutto del gerundio assoluto e sulla collocazione del soggetto (217-221) - e in Goidànich (1919); a proposito dei pronomi personali, il glottologo segnala che «Nel gerundio composto il pron. pers. sogg. si pone di preferenza tra l'ausiliare e il participio: Avendo tu mancato di parola; Avendo mancato tu di parola può essere interpretato anche come un'espressione enfatica» (ivi: 155).

Molte delle possibilità distributive e funzionali che il gerundio ha conosciuto lungo la storia della lingua italiana e dei suoi dialetti sono invece state illuminate in tempi più recenti dagli studiosi: dalla iniziale concorrenza col participio circostanziale (frase relativa) del gerundio strumentale-modale, declinato all'ablativo (Viridis, 1983), al gerundio in funzione di imperativo comune nei dialetti meridionali estremi (Rohlfs, 1969: \ 722), alla sovrapposizione con l'infinito (De Roberto, 2013: 878), alla possibile anteposizione del soggetto in una subordinata gerundiva (Egerland, 2010; Salvi, Renzi, 2010-2011: 8), ai gerundi retti da preposizioni (Rohlfs, 1969: \ 721; Egerland, 2010; Salvi, Renzi, 2010-2011: 28) o concordati con l'oggetto (Egerland, 2010; Dardano, 2012 e 2020), sino a specializzazioni di singoli tipi semantici del gerundio in precise tipologie testuali, come i testi argomentativi e narrativi del XIII e XIV (Frenguelli, 2003).

Analogamente le recenti grammatiche di consultazione compiono un'analisi approfondita, discriminando sempre le sfumature semantiche che può sussumere questo modo verbale (Salvi, Vanelli, 2004; Schwarze, 2009; Serianni, 2000; Renzi, Salvi, Cardinaletti, 1988-1995) e approfondendo i vincoli più strettamente sintattici (distribuzione rispetto alla reggente; coreferenzialità tra soggetto del gerundio con una delle funzioni del verbo della frase matrice: possibilità del gerundio assoluto: Salvi, Vanelli, 2004: 245-246; Schwarze, 2009: 166), anche in diacronia (si veda la desuetudine segnalata da Schwarze, 2009: 166 a proposito dei costrutti coreferenti con l'oggetto).

A proposito dell'omissione del soggetto nelle frasi gerundive, ai fini della presente ricerca è degna di menzione la possibilità che il gerundio coordinato sia controllato «dall'evento espresso nella frase matrice, come in (8), dove il Soggetto del gerundio non è 'la banca' o 'gli svaligiatori', ma 'lo svaligiamento della banca': (8) La banca era stata svaligiata, lasciando i risparmiatori nella miseria più totale» (Salvi, Vanelli, 2004: 246). La possibilità del gerundio di non essere controllato da un referente anaforico puntualmente espresso a livello superficiale di frase, bensì da un attante inespresso ma ricavabile dal significato della proposizione reggente, rappresenta l'anello di congiunzione tra la modalità sintattica e la modalità semantico-pragmatica di gestione e distribuzione del gerundio, cui possono agganciarsi le note e già menzionate derive sintattiche degli scriventi inesperti (vd. infra par. 5.5).

A fronte della più recente e più esaustiva sistematizzazione dell'argomento, il parlante mediamente istruito spesso memorizza soltanto una delle prescrizioni; non sono incolpevoli le «spiegazioni approssimative di molti libri di grammatica (desiderosi di essere soprattutto brevi)» (Sabatini, 2009), che citano per lo più l'obbligo di coreferenzialità del soggetto tra il gerundio subordinato e la sua reggente (pure contravvenuto nella pratica: cfr. par. 5.5). Ancor meno interiorizzato, anche per il suo carattere più ricercato ed estraneo al neostandard, è un corollario alla regola, secondo il quale «il gerundio di una frase implicita 
può riferirsi anche a un soggetto diverso da quello della reggente a patto che quel soggetto venga introdotto, con un nome o un pronome» (ibid.) coll'accorgimento di posporre il soggetto nella frase col gerundio. Anche gli altri casi di non "coreferenza", pur vivi nella pratica dei nativi (cfr. par. 4), sono più facilmente rimossi dalla propria consapevolezza metalinguistica:

quando si mette al gerundio un verbo impersonale (Piovendo a dirotto, non siamo usciti di casa); quando si introduce un cosiddetto "soggetto generico" (Ripensandoci, le tue parole non mi sono piaciute; cfr. L. Renzi, Grande grammatica italiana di consultazione, vol. II, Bologna, Il Mulino, 1991, pp. 572-574), meglio ancora quando un soggetto generico del gerundio si associa a una reggente impersonale: (sbagliando, s'impara). (ibid.; cfr. Serianni, 2006/2017: 170-171)

Del resto, a scuola, lo studente può ricevere messaggi contrastanti sull'opportunità d'impiegare il gerundio: se da un lato negli scritti di italiano si incentiva l'uso di proposizioni esplicite, più trasparenti in virtù dell'espressione dei rapporti logici tramite i connettivi, dall'altro lato per le traduzioni delle versioni latine se ne incoraggia il ricorso quando si è in dubbio sul significato logico-semantico della proposizione da tradurre: «il gerundio non sarà magari la soluzione ideale, ma difficilmente sarà sbagliato» (Cortelazzo, 2014).

Proprio la grande duttilità e ambiguità espressiva del gerundio (Barattella, 2015-2016), in buona parte dovute ai molteplici valori assumibili, consente allo scrivente meno esperto di costruire abbastanza agilmente periodi ipotattici di una certa lunghezza (vd. infra par. 4): il gerundio è una sostituzione economica in luogo di paratassi o ipotassi, che consente di non soffermarsi a riflettere su quale connettivo impiegare (Solarino, 1991); potendo esprimere anche la posteriorità, il gerundio semplice può infatti sostituire anche la coordinazione, rivelandosi una comoda strategia paraipotattica, non molto dissimile dal gerundio usato agli albori della prosa italiana (Solarino, 1992). Anche per gli studenti universitari del nuovo millennio il gerundio risulta essere «la forma implicita di gran lunga preferita [...] per ovviare alle difficoltà di coesione che presentano le strutture esplicite» (Gualdo, 2010: 40).

\section{IL CORPUS DI SCRITTI}

Il corpus di indagine consta di 413 elaborati scritti così ripartiti:

- 181 scritti, eterogenei per tipologia testuale e per estensione, prodotti nel liceo scientifico G. Carducci di Milano negli anni 2011 (18 scritti di una classe seconda) e 2012 (18 scritti di una classe prima, 44 di una classe seconda, 18 di una classe terza, 83 di una classe quinta - comprensivi di alcune simulazioni dello scritto di maturità $)^{4}$;

- 121 scritti (riassunti di un articolo di giornale, con un'estensione compresa tra le 250 e le 350 parole) prodotti nel 2018 nelle classi prime degli istituti superiori di Oggiono e di Monticello Brianza (LC), con diverso indirizzo di studio (liceo linguistico, liceo delle

\footnotetext{
4 Ringrazio la professoressa Ilaria Bonomi per avermi donato questo corpus.
} 
scienze umane, istituto tecnico informatico e delle telecomunicazioni, istituto tecnico chimica e materiali) ${ }^{5}$;

- 111 elaborati (testi argomentativi sul modello del Five Paragraph Essay, con una lunghezza massima di 720 parole) prodotti all'Università degli Studi di Milano come prova finale dei laboratori telematici di lingua e scrittura italiana; 22 scritti sono stati prodotti durante l'a.a. 2018/2019, 89 durante l'a.a. 2020/2021. Gli scriventi afferiscono a diversi corsi di laurea triennali a indirizzo umanistico (Filosofia, Lettere, Lingue e letterature straniere, Scienze della comunicazione, Scienze storiche).

Per quanto il corpus non sia ben equilibrato, soprattutto per la rappresentanza quantitativamente difforme delle cinque classi di scuola secondaria, si ritiene che possa ugualmente offrire del materiale per riflessioni significative.

I dati, ottenuti attraverso uno spoglio manuale, verranno confrontati con le tendenze ricavate dai corpora on line VINCA (Varietà di Italiano di Nativi Corpus Appaiato: http://www.valico.org/vinca_CORPUS.html) e, in alcuni casi, VALICO (Varietà Apprendimento Lingua Italiana Corpus Online: http://www.valico.org/valico.html), per le coorti approssimativamente ${ }^{6}$ corrispondenti a quelle considerate nel presente studio (14-18 e 19-25 anni).

Si avverte, infine, che i campionamenti estrapolati dal corpus sono riprodotti nella loro facies originale, anteriore alle correzioni dei docenti e comprensiva di tutti gli errori inerenti ai diversi livelli linguistici.

\section{VITALITÀ DEL GERUNDIO SUBORDINATIVO}

La tabella che segue mostra l'incidenza dei gerundi subordinativi all'interno del corpus, sia in termini percentuali che di mero computo numerico. Si consideri che uno stesso elaborato può contenere tanto gerundi corretti (almeno uno) aderenti allo standard o agli usi neostandard, quanto gerundi agrammaticali; elaborati simili sono quindi stati conteggiati per entrambi i gruppi "senza errori" e "con errori”. La fenomenologia indagata non include la perifrasi aspettuale stare + gerundio in costrutti subordinati impliciti, pure interessata da cambiamenti nell'italiano contemporaneo (Rossi, 2009).

Il prospetto documenta come il gerundio subordinativo sia ancora vitale negli scriventi delle scuole superiori: in buona parte degli scritti è possibile rintracciare almeno una occorrenza di gerundio subordinato, usata correttamente. Se le classi prime fanno registrare le percentuali ${ }^{7}$ inferiori al 60\% di incidenza (55\% nel 2012, 57\% nel 2018), gli elaborati con almeno una subordinata gerundiva sono in media il $66 \%$ degli scritti totali. Il gerundio subordinato gode di ottima salute negli elaborati universitari, nei quali si manifesta in media nel 90\% degli scritti. Si può quindi affermare che in generale il gerundio subordinativo è ben saldo negli usi scrittori delle generazioni più giovani; di seguito si vedrà in quali termini si concretizzi questa vitalità.

\footnotetext{
5 Sul presente corpus, punto di partenza per la pianificazione di un Programma Operativo Nazionale (PON) per la scuola, si veda anche Dota, Prada (2020).

${ }^{6}$ L'avverbio è d'obbligo considerando i (pochi) frequentanti dei laboratori di lingua e scrittura italiana più adulti.

${ }^{7}$ Le percentuali sono arrotondate alla cifra più vicina al risultato matematico.
} 
(C) Italiano LinguaDue 2. 2021. M. Dota, L'uso delle subordinate gerundiali nella scrittura scolastica e universitaria

Tabella 1. Incidenza delle subordinate gerundiali nel corpus

\begin{tabular}{|l|l|l|l|l|}
\hline \multicolumn{5}{|c|}{ Presenza del gerundio subordinativo nel corpus } \\
\hline Classi & Scritti totali & $\begin{array}{c}\text { Senza errori } \\
\text { (almeno 1. g) }\end{array}$ & $\begin{array}{c}\text { nessun } \\
\text { gerundio }\end{array}$ & \multicolumn{1}{|c|}{ Con errori } \\
\hline $2^{\wedge}$ sup. 2011 & 18 & $12(66 \%)$ & $4(22 \%)$ & $2(11 \%)$ \\
\hline $1^{\wedge}$ sup. 2012 & 18 & $10(55 \%)$ & $6(33 \%)$ & $2(11 \%)$ \\
\hline $2^{\wedge}$ sup. 2012 & 44 & $28(63 \%)$ & $12(27 \%)$ & $4(9 \%)$ \\
\hline $3^{\wedge}$ sup. 2012 & 62 & $40(64 \%)$ & $16(25 \%)$ & $8(12 \%)$ \\
\hline $5^{\wedge}$ sup. 2012 & 83 & $60(72 \%)$ & $19(22 \%)$ & $14(16 \%)$ \\
\hline $1^{\wedge}$ sup. 2018 & 121 & $70(57 \%)$ & $43(35 \%)$ & $17(14 \%)$ \\
\hline Lab. Scrit. 2018-19 & 22 & $20(90 \%)$ & $2(9 \%)$ & $2(9 \%)$ \\
\hline Lab. Scrit. 2020-21 & 89 & $73(82 \%)$ & $5(5,6 \%)$ & $11(12,3 \%)$ \\
\hline
\end{tabular}

Nella valutazione non bisogna però trascurare l'eventualità che la tipologia testuale elicitata possa aver condizionato le probabilità di emersione del gerundio: se si considerano gli elaborati nel complesso, emerge come i gerundi subordinati scarseggino nei temi costruiti secondo il modello dell'articolo di cronaca giornalistica e nelle analisi dei testi letterari (in particolare, nelle simulazioni della prima prova di maturità, che possono superare per estensione i testi degli altri due subcorpora); viceversa, abbondano nei temi e saggi a carattere argomentativo ed espositivo, compresi i temi che vertono sugli argomenti di letteratura (come il tradizionale confronto dei tre principali personaggi clericali de I promessi sposi), più consueti a partire dalla classe terza superiore. Coerentemente con il curricolo, le tipologie testuali esercitate nelle classi inferiori sono invece descrizioni e narrazioni di episodi personali, anche in forma epistolare o, come si è detto, riassunti di un testo (articolo giornalistico) dal carattere prevalentemente espositivo.

Riguardo all'assenza di subordinate al gerundio, si vede come le percentuali più elevate riguardino gli scriventi delle classi prime (33\% nel 2012 e 35\% nel 2018), le medesime a far registrare le percentuali più alte per gli errori di accordo morfosintattico. Questo assetto suggerisce che questa fase scolastica sia cruciale per lo sviluppo nell'apprendimento e consolidamento dell'uso del gerundio subordinativo. È infatti altrettanto significativo che negli scritti delle prime classi di scuola superiore - ma non solo - si concentri la gran parte dei periodi la cui subordinazione è affidata a catene coordinate, spesso asindetiche, di gerundi (documentate anche in scriventi più adulti: cfr. Gualdo, 2010: 44 e, per gli scritti dei maturandi, Ruele, Zuin, 2020: 182):

$\left(2^{\wedge} \mathrm{S}, 2012\right) \quad$ La posizione di Manzoni a riguardo la esprime nel libro analizzando personaggi appartenenti alla parte negativa della chiesa (Don Abbondio, la monaca di Monza) non dimenticandosi però delle figure appartenenti al lato positivo (Fra Cristoforo, il cardinale Federico Borromeo) rammentandoci l'importanza della chiesa nella nostra società 
(3^S, 2012) loro vivono rispettando un proprio codice di leggi, pensando solo al proprio bene, non rispettando alcuna regola, creando una propria economia $[\ldots]$ e minando quindi $[\ldots]$

(5^S, 2012) Non ancora adulto inizia a far parlare la terrà di sé, andando contro al padre mercante facendo, pubblicamente, voto di povertà spogliandosi in presenza del padre e della corte ecclesiastica.

$\left(5^{\wedge} S, 2012\right) \quad$ Le scienze speculative hanno presto avviato indagini conoscitive con metodo e rigore intellettuale, suddividendo e classificando, ponendosi precisi quesiti e tentando di dare altrettanto precise risposte sviluppando così dottrine, culti $[\ldots]$

$\left(1^{\wedge} \mathrm{S}, 2018\right) \quad$ Si tende ha mostrare ciò che si vuole essere una selettiva, postando solo determinati argomenti oppure postando tutta la propria vita privata, a volte postando cose che non dovrebbero essere viste dai parenti, allontanando il contatto.

Talvolta la tendenza a subordinare attraverso una serie di gerundi fa sì che lo scrivente perda il filo della coesione testuale, non ricordando di aver mutato il soggetto nel corso del dettato e incappando quindi in errore:

$\left(5^{\wedge} \mathrm{S}, 2012\right) \quad$ Lo stesso presidente fornisce alcuni dati [articolo sulla Repubblica 1 aprile 2011] in percentuali, riguardanti il tipo di alimentazione seguito dalla popolazione italiana, affermando che il $95 \%$ dichiara che il pasto più importante è il pranzo, ma nonostante tutto abusa di carboidrati $\mathrm{e}$ carni grasse, tralasciando quelle magre, pesce e verdure, spiegando infine l'importanza di questi alimenti

I dati ricavabili dalle trascrizioni dei testi orali prodotti da parlanti nativi per il corpus VINCA confermano la tendenza. In proposito, non bisogna trascurare la percezione generale degli studenti rispetto a questo stilema: la «moltiplicazione dei gerundi» rientri tra i segnali e le marche linguistiche «percepiti come tipici dello scritto» (Ruele, Zuin, 2020: 182) diafasicamente sostenuto (cfr. Salvatore, 2019: 67). Appare quindi evidente che lo scritto formale di riferimento sia ancora quello burocratico-giuridico-amministrativo, l'unico che esibisce la medesima abbondanza e seriazione di gerundi, per quanto tendenzialmente vincolati alla porzione incipitaria del testo (Trifone, 2006/2009).

La duttilità di questo modo, e la sua opacità semantica, infatti, consentono di operare una subordinazione meno meditata, ma al contempo possono facilmente indurre gli scriventi in errore. L'esercizio dell'analisi del periodo, tradizionalmente svolta al terzo anno della scuola secondaria di primo grado e ripetuta nel biennio delle scuole superiori, sembra avere poche ricadute nel breve periodo sul miglioramento d'uso di questa struttura linguistica e sulla generale capacità di subordinazione. In generale, negli elaborati di prima superiore predomina infatti la coordinazione.

Gli slogamenti morfosintattici dei gerundi subordinati (considerati nel par. 5.5) confermano come in questa fase gli scriventi, pur avendo riflettuto e riflettendo metalinguisticamente sull'ipotassi e sulla classificazione semantica delle subordinate, per quanto concerne alcune tipologie di proposizioni (come le relative, ugualmente critiche in 
diversi elaborati), prediligano la modalità semantico-pragmatica di strutturazione della frase. Come accade per gli apprendenti di una L2, se non esposte ad input linguistici e feedback correttivi adeguati, tali abitudini, non essendo percepite come errate, si fossilizzano: lo conferma il persistere dei medesimi errori nelle scritture dei giovani adulti, sebbene l'incidenza delle forme errate sia nettamente ridotta.

\section{USI DEL GERUNDIO}

Nei paragrafi che seguono sono considerati singolarmente gli usi documentati del gerundio, standard, neostandard e aberranti.

\subsection{Tipi semantico-nozionali prevalenti e collocazione delle gerundiali nel periodo}

Nel corpus è dominante il gerundio con sfumatura modale (strumentale o di maniera), soprattutto tra gli scriventi più giovani, che ricorrono anche al tipo temporale. Un assetto analogo è riscontrabile nel corpus VINCA (2004), per le trascrizioni dei testi orali descrittivi (198) di italofoni nativi frequentanti le scuole superiori: la maggior parte delle subordinate gerundiali è per lo più di carattere modale. Questa sfumatura semantica domina ugualmente nei testi orali prodotti da studenti universitari del medesimo corpus (428), nei quali affiorano inoltre le subordinate gerundiali causali.

Analogamente nel nostro corpus le gerundiali causali occorrono maggiormente tra gli scriventi più maturi e costituiscono la quarta tipologia semantica prevalente; le gerundiali causali sono spesso incidentali, ma spesso tale collocazione sintattica non è precisata dalla punteggiatura:

$\left(2^{\wedge} S, 2012\right) \quad$, ma che essendo un sentimento molto profondo vada maturato nel tempo.

Ormai essendo nel terzo millennio bisognerebbe avere la parità

$\left(3^{\wedge} S, 2012\right) \quad$ Le immagini avendo un'importanza maggiore vengono $[\ldots]$

Inoltre essendo l'acqua l'elemento della vita in mancanza di essa, i campi non sarebbero più irrigati

$\left(5^{\wedge} S, 2012\right) \quad$ Anguilla $[\ldots]$, non avendo conoscenza [...], dopo aver fatto fortuna in America torna nel luogo [...]

Essendo un trovatello Anguilla non sa chi sono i suoi genitori

e il protagonista non sapendo la propria non sa che valore o identità darà

[...] poiché essendo gli esseri umani uguali devono avere uguali diritti

[...] poiché essendo luoghi della sua infanzia lui ritiene che gli appartengono 
e lo stesso autore sostiene che, non esistendo ancora una teoria accettata sullintelligenza generalista, la distanza temporale che ci separa da uno sviluppo pratico è «maggiore di quella tra l'attuale trasporto spaziale e i viaggi a velocità maggiore della luce», perché di questi ultimi abbiamo già teorie largamente condivise nel mondo accademico.

Avendo poi poco tempo a disposizione per far passare un'idea, la pubblicità utilizza $[\ldots]$

(U 2020/21) Il servizio di Substack è inoltre difficile da classificare, essendo una società di software che, imitando alcune delle funzioni delle redazioni, produce un eccesso di mail [...]

Quando si tratta di economia, infatti, non è sufficiente guardare fuori da noi, raccogliere dati e stipulare teorie, perché trattandosi di esseri umani, è dentro di noi che dovremmo guardare.

Essendo di natura elettronica, i libri digitali hanno una replicabilità potenzialmente infinita

lo schermo, rispondendo ai nostri comandi, ci chiude nell'unico mondo su cui pensiamo di avere potere

Piuttosto fortunato è il tipo consecutivo, che appare perlopiù in combinazione con l'avverbio così, principalmente nelle scritture scolastiche:

$\left(2^{\wedge} S, 2011\right) \quad$ Problemi di questo genere al mondo però che ne sono molti $[\ldots]$ che hanno portato la distruzione di gran parte della vegetazione terrestre, togliendo così posti di vista animale

l'uomo occidentale è riuscito a capire il vantaggio nel dare alle donne la possibilità di esplicare le proprie capacità portando così a delle innovazioni in tutti i campi della società moderna. [...] non credono che esistano questi avvenimenti prendendo così, secondo me, ingiro la dignità della donna.

dovevano raggiungere il prete, costringendolo, così, a dargli

$\left(2^{\wedge} \mathrm{S}, 2012\right) \quad$ Le violenze che hanno subito e la tragica morte che hanno fatto hanno causato molto scalpore, facendo sì che le massime cariche dello stato parlassero del fenomeno e cercassero soluzioni per arginarlo.

(3^S, 2012) L'uomo viveva in piccoli villaggi riunendosi spesso con altre persone [..], formando così una comunità

Continua suo malgrado ad infrangere le leggi, distruggendo così un'armonia

Sposò la povertà, cominciando così la sua vita religiosa.

$\left(5^{\wedge} S, 2012\right) \quad$ Spesso le piazze sono mezzi di propaganda e manifestazioni artistiche e raccolgono [...], favorendo uno scambio culturale

Le scienze speculative hanno presto avviato indagini conoscitive con 
metodo e rigore intellettuale, [...] sviluppando così dottrine, culti

quest'ultimo metodo stimola maggiormente lo scambio di opinioni e i dibattiti, creando così un'interazione tra ogni individuo

Egli si trascurava, diventando così cieco

Fortunatamente vi sono sempre delle eccezioni, persone che $[\ldots]$ riescono a trasmetterci un messaggio, acquistando così una fama positiva, genuina e non malsana.

È, però, vero che la società è bombardata di pubblicità che tendono a pubblicizzare prodotti di scarsa qualità e tende a fare accostare il valore delle cose al loro costo, costruendo l'ideale che più una cosa costi, più valga.

$\left(1^{\wedge} \mathrm{S}, 2018\right) \quad$ La presentazione può avvenire in modo selettivo oppure condividendo qualsiasi cosa perdendo la consapevolezza che il materiale può esser visto da tutti

L'adesione ad un social permette di soddisfare il bisogno di appartenenza e di affermare se stessi, comunicando così al mondo esterno una falsa visione di noi

, invece, si tende a generalizzare, in modo da creare un profilo accessibile a tutti, perdendo così comunicazioni mirate

Gli utenti tendono a esporre sul profilo la propria rappresentazione ideale, diventando così ciò che desiderano essere.

(U
La distribuzione di un apparecchio elettronico impegna diversi [sic] vie per arrivare a destinazione, producendo così emissioni da carburanti e derivati.

I tipi concessivo e ipotetico, invece, sopravvivono tra gli scriventi universitari e le classi superiori della scuola secondaria, del resto chiamate a produrre più delle altre classi testi di carattere argomentativo, nei quali le mosse comunicative corrispondenti (fare una concessione o dichiarare una condizione) sono senz'altro più implicate che nei testi descrittivi e narrativi. Una conferma ulteriore è fornita dal corpus KIParla, nel quale le occorrenze di gerundi di tipo ipotetico occorrono quasi esclusivamente nel teacher talk di argomento scientifico, come nell'esempio:

vi ricordate che la probabilita' non e' nient' altro che il limite della frequenza relativa / immaginando di andare a fare un numero infinito di campionamenti // quello che succede e' che ciascuna //

Il carattere espositivo argomentativo del testo originale da riassumere fa sì che nel nostro corpus sia possibile rilevare usi gerundiali concessivi anche in alcuni scritti della classe prima. Di seguito si riportano le occorrenze totali riscontrate: 
CONCESSIVO

$\left(2^{\wedge} \mathrm{S}, 2012\right) \quad$ e non accetto che avendo lo stesso posto di lavoro la donna debba guadagnare meno

Pur essendosi dimesso non cancellerà il suo errore

$\left(3^{\wedge} \mathrm{S}, 2012\right) \quad$ pur essendo state affrontate in epoche precedenti sono questioni molto attuali

Ma, Tommy pur nutrendo grande simpatia per Kathy, si fidanza con Ruth

$\left(1^{\wedge} S, 2018\right) \quad$ Sui social network, pur essendo libera la scelta dei post da aggiungere al profilo, risulta essere [...]

Pur essendo un paradosso, viene definito uno strumento utile

esse pur sapendo che Facebook [..] è una piattaforma dove vengono mostrati gli spazi più intimi di se stessi, si è verificato che $[\cdots]$

(U 2018/19) questo è riconosciuto implicitamente anche da chi, pur non sostenendo il loro diritto a non essere sfruttati e uccisi, supporta il metodo della sperimentazione animale o anche solo si serve dei prodotti testati grazie a tale metodo

(U 2020/21) Ebbene, pur non negando i problemi sovraesposti, possiamo davvero trovare nella pena capitale la soluzione a questi mali?

Pur non potendo negare il fascino del libro cartaceo, è bene chiedersi, soprattutto in questi anni, se la strada da percorrere sia di cieco egoismo oppure di altruismo verso l'ambiente

Pur partendo dallo stesso contesto che produce le avanguardie, non cercano la rottura con la tradizione

\section{IPOTETICO}

$\left(3^{\wedge} S, 2012\right)$

$\left(5^{\wedge} S, 2012\right)$

Andando avanti di questo passo si avrà sempre più una cultura [...] Nel momento in cui, facendogli ascoltare una canzone mi risponde "che schifo"

Volendo riassumere il testo di Cesare Pavese, La luna e i falò, potremmo

(U 2020/21) Analizzando la presenza degli Stati Uniti nel mondo possiamo capire fin dove la sua influenza arrivi

Analizzando il "caso Apple" sorgono spontanee alcune domande:

Guardando infatti ai nuovi canali con cui normalmente interagiamo, notiamo come l'esprimere la propria opinione a tutto il mondo usando, per ironia della sorte, un telefono, 
Stando a quanto dichiarato dal presidente della Toyota Akio Toyoda in una sua conferenza, le centrali elettriche non sono attualmente in grado di fornire energia ad un ipotetico parco auto completamente elettrico.

Leggendo i commenti in particolare sotto le foto degli influencer, possiamo notare la grande quantità di insulti riferiti all'aspetto fisico,

È una spesa enorme considerando che è solo una parte di quella totale fatta dai tifosi

Per quanto concerne la distribuzione del gerundio nel periodo, prevalgono le collocazioni agli estremi, dunque incipitarie (come in parte già visto) o in chiusura di periodo. Tale collocazione, attestata in tutti i gradi di istruzione considerati, gode da discreto tempo di ampia fortuna: riferendosi a elaborati universitari, Gualdo (2010: 44) rileva la ricorsività della «struttura che si serve di una gerundiale conclusiva per evitare una coordinazione (o un connettivo esplicito) che porrebbe problemi di accordo morfosintattico. In periodi non molto complessi il gerundio pare quasi svolgere il ruolo di "demarcativo" di fine periodo». Di seguito si riportano alcuni campionamenti dal nostro corpus:

$\left(2^{\wedge} S, 2011\right) \quad$ Per cacciare la tigre, l'uomo si inoltra nella foresta di notte, mettendo a rischio la propria vita.

Ha imposto la sua autorità agli altri esseri viventi senza preoccuparsi delle conseguenze e ora modifica il ciclo vitale uccidendo parte di esso.

[...] che poi ognuno avrebbe ripetuto al compagno dando un espressività alla parola.

$\left(3^{\wedge} \mathrm{S}, 2012\right) \quad$ i genitori decidono di approvare pensando che finalmente loro figlio stesse iniziando a socializzare creandosi qualche amico

$[\ldots]$ che appare nel sogno per via del subconscio, interferendo con l'operazione.

$\left(5^{\wedge} \mathrm{S}, 2012\right) \quad$ Ungaretti in questa poesia espone il tema del sentimento del tempo in maniera esaudiente, sottolineando quindi come la vecchiaia impedisca di esaudire desideri repressi.

$\left(1^{\wedge} S, 2018\right) \quad$ Uno stesso racconto poteva essere espresso a persone diverse, in modo differente a seconda della persona alla quale voleva essere raccontato, mettendo in evidenza fatti diversi.

In tanti creano il loro profilo attendendosi, non più alla realtà, ma a come uno vorrebbe essere, nascondendo la verità tramite la rete. $\mathrm{Si}$ trova così, nei social, la possibilità di migliorare il proprio aspetto e la propria persona, manipolando la realtà e ingannando gli altri.

Si può evincere pertanto che aderire ad un social significa l'aver bisogno di affermare se stessi e sentirsi parte di qualcosa; ciò fa si che gli utenti siano spinti a mostrare un loro sé ideale piuttosto che 
mostrarsi per come sono selezionando con cura i propri post.

(U 2018/19) Queste nuove possibilità hanno modificato il nostro modo di vivere la fiducia, estendendola e diffondendola.

Diverse aziende stanno ampliando il loro pensiero, eliminando i luoghi comuni proponendo messaggi positivi.

(U 2020/21) L'attività giornalistica sta cambiando il suo modo d'essere, investendo sempre di più sulla rete e abbandonando pian piano il mondo cartaceo.

Il paternalismo politico è un atteggiamento per il quale i governanti agiscono dispoticamente ma garantendo il benessere ai governati, non ritenendoli capaci di perseguirlo autonomamente.

La disparità numerica tra giornalisti professionisti e pubblicisti iscritti all'albo è una diretta conseguenza delle difficoltà di accesso alla professione, rappresentando di fatto una delle maggiori criticità della disciplina.

Le situazioni emozionali e i dilemmi morali, che sono il cosiddetto "materiale letterario", consistono anche in un valido esercizio per il cervello incoraggiandoci verso le menti di personaggi immaginari, incrementando la nostra capacità empatica nella vita quotidiana.

La bolla tuttavia è costruita anche da ognuno di noi qui che tende ad interagire principalmente con chi ha opinioni simili, autoalimentando la propria visione del mondo, eliminando da questa prospettiva $\mathrm{i}$ punti di vista alternativi, e fomentando la credenza che la maggior parte delle persone la pensi alla nostra stessa maniera.

Ma questa piaga non è incontrastabile se lo si vuole, e vi si può porre fine: rimediando al passato, informando e coinvolgendo più persone possibile nel presente, e prevenendo un pericoloso futuro.

Quando poi lo scrivente chiosa l'intero elaborato con una gerundiale, ottiene l'effetto di mostrare di avere chiaro «il quadro della situazione» (Serianni, 2013: 10). Tale giacitura ricorre significativamente negli scritti di maturità (Ruele, Zuin, 2020: 155) e si conferma tra le preferenze degli scriventi di scuola superiore del nostro corpus:

$\left(2^{\wedge} \mathrm{S}, 2011\right) \quad$ Le parole di questo inventore, in ogni caso, non sono da tenere in considerazione solo adesso, quanto in futuro poiché le persone dovrebbero seguire i suoi consigli togliendosi ogni limite dalla testa arrivando a raggiungere anche gli obbiettivi al limite dell'impossibile.

$\left(3^{\wedge} S, 2012\right) \quad$ Con la nostra morte si perderanno le nostre storie, quindi penso che non bisogna mai perdere l'occasione di raccontarle, in modo che vengano ricordate, ed una parte di noi stessi rimarrà nel tempo, sperando che le storie non muoiano mai.

Continua suo malgrado ad infrangere le leggi, distruggendo così un'armonia. 
[...] altrimenti confondono solamente, trasmettendo messaggi sbagliati come quello della donna oggetto.

$\left(5^{\wedge} \mathrm{S}, 2012\right) \quad$ Ma la piazza rimarrà sempre un luogo della memoria, rieccheggiando l'intera storia [...], rimanendo inalterata nel tempo e offrendo sempre occasioni di incontro.

Le aperture gerundiali, invece, hanno spesso carattere testuale o metatestuale, soprattutto tra gli scriventi di scuola superiore, come già rilevato da Ruele e Zuin per i maturandi (2020: 154); il gerundio presente incipitario può diventare quindi il segnale di apertura di un nuovo movimento testuale o del pensiero verbalizzato:

$\left(2^{\wedge} \mathrm{S}, 2012\right) \quad$ Tralasciando il mondo del lavoro e il passato, le donne hanno $[\ldots]$

Riflettendo però come sarebbe la vita senza un opposto alla nostra personalità?

(3^S, 2012) Partendo dal significato etimologico della parola "filosofia" si può giungere a

Quindi ricollegandoci ad Aristotele [...]

Per esempio, citando la situazione attuale, circa il 50\% della popolazione italiana non paga le tasse

Rispondendo alla domanda di Montale, io penso che

(5^S, 2012) Passando ad una analisi del testo si nota che [...] Procedendo nella lettura notiamo che [...] Ponendo sempre come base del discorso il passato, Anguilla, riflette sui nomi prendendo in considerazione solo l'aspetto materiale, definendoli: "carni".

Tralasciando la questione dello sfruttamento dei lavoratori nelle fabbriche, molte persone non sono d'accordo nel sostenere la positività di questa rivoluzione

Pensandoci bene è giusto connettersi

$\left(1^{\wedge} S, 2018\right) \quad$ Confrontando i tempi precedenti a Facebook e quelli moderni, è possibile notare che $[\ldots]$

(U 2018/19) Tralasciando i programmi dediti al mero intrattenimento personale, i telegiornali ed i canali di informazione hanno un valore inestimabile [...] Non calcolando il costo iniziale del mezzo (che va dal più economico del quotidiano alla tv schermo piatto di ultima generazione) riceviamo servizi che non paghiamo

$\mathrm{Ma}$, andando nello specifico, questi studiosi hanno compreso che $[\ldots]$

(U 2020/21) Prendendo in considerazione per l'anno 2020 le classifiche dei libri più venduti e le classifiche delle ultime novità pubblicate settimanalmente sui quotidiani, si riscontrano diverse somiglianze.

Ripercorrendo gli argomenti del testo potremmo affermare che la 
nascita della bubble democracy, si identifica in un sistema sociale,

Partendo dalle considerazioni precedenti, sembra necessario un cambio di rotta che riporti l'individuo in possesso di se stesso e della storia

Facendo un passo indietro di qualche secolo, oltre che delle distinzioni di classe, le diete rappresentavano anche delle distinzioni patriarcali

Partendo dall'analisi di tre casi, si risalirà poi alla radice del problema

Nel loro rapporto di ricerca i due docenti riconoscono «la quasi grammaticalizzazione di espressioni come concludendo». Nel nostro corpus i vocaboli concludendo e simili sono rari; si registrano soltanto tre esempi:

$\left(3^{\wedge} S, 2012\right) \quad$ Concludendo l’uomo per Aristotele è $[\ldots]$

(U 2018/19) Concludendo, i problemi legati alle AI di cui dovremo sicuramente occuparci, come la competizione con il lavoro umano e i risvolti etico-economici, sono molti.

(U 2020/21) Concludendo, ritengo che la possibilità di rendere l'editoria digitale una valida alternativa a quella cartacea dipenderà dalla nostra attitudine al mezzo.

Il gerundio presente è incluso più di frequente in strutture analitiche dalla funzione testuale equivalente, quali concludo dicendo:

(3^S, 2012) Aristotele conclude dicendo

Voglio concludere dicendo che la musica [...]

$\left(3^{\wedge} S, 2012\right) \quad$ Volendo riassumere il testo di Cesare Pavese, La luna e i falò, potremmo $[\ldots]$

Concludo dicendo che in ogni ambiente ed occasione il più forte dominerà il più debole come recita perfettamente l'affermazione di Sartre.

E conclude dicendo che $[\ldots]$

\section{Concludo dicendo}

Il gradimento per i segnali discorsivi gerundiali con funzione metatestuale emerge altresì dalla loro occorrenza, seppur minore, all'interno del periodo:

$\left(3^{\wedge} \mathrm{S}, 2012\right) \quad[\ldots]$ ho notato che in alcuni comuni o comunque generalizzando nell'hinterland che sono rimasti alcuni paesini legati alle tradizioni

$\left(5^{\wedge} \mathrm{S}, 2012\right) \quad[\ldots]$ ovvero trovare una casa e una terra in cui stanziarsi e, riprendendo una frase del testo, fare in modo che [...] 
Sempre in attacco di periodo è possibile trovare gerundi modali di tipo inclusivo; il gerundio assume quindi valore aspettuale imperfettivo rispetto al valore perfettivo espresso dalla reggente successiva:

(3^S, 2012) Oggigiorno ascoltando l’ipod di una persona per la maggior parte delle volte si capisce più o meno che persona sei $[\ldots]$

Combattendo la criminalità difende tutta quella parte di società,

$\left(5^{\wedge} S, 2012\right) \quad$ Dandoci tanti nomi riesce ad esprimerci $[\ldots]$

Nominandoli con i loro nomi propri, vuole stare a sottolineare il fatto che $[\ldots]$

Girando il mondo e viaggiando Anguilla percepisce che $[\ldots]$

$\left(1^{\wedge} S, 2018\right) \quad$ Stabilendo un profilo si soddisfano due bisogni

Anche i gerundi temporali che esprimono coincidenza rispetto alla reggente possono essere collocati all'inizio del periodo:

$\left(1^{\wedge} S, 2018\right) \quad$ Perdendo il potere nella gestione delle proprie informazioni si acquista facilità di accesso

Perdendo il potere delle proprie informazioni se né aquista per accedere a quelle degli altri.

\subsection{Uso iconico del gerundio presente}

Sempre in apertura di periodo, secondo un ordine di collocazione degli eventi o stati espressi di tipo iconico, abbondano $i$ gerundi presenti che esprimono un rapporto di anteriorità rispetto alla reggente. Già negli scritti scolastici analizzati da Solarino (1992: 17) l'ordine iconico era rispettato nella quasi totalità dei casi: sul totale degli elaborati «solo 16 (il $6,3 \%$ ) presentano infatti un ordine inverso a quello cronologico: evidente segnale della maggiore difficoltà di uso di questi ultimi» (ibid.).

Nel nostro corpus quell'assetto trova piena conferma e validità per tutte le classi di età; non si registrano, anzi, casi di ordine inverso rispetto a quello cronologico. Di seguito si forniscono alcuni specimina:

$\left(2^{\wedge} \mathrm{S}, 2012\right) \quad$ vedendo che i bravi non cambiavano idea, obbedisce agli ordini di Don Rodrigo perché, non riuscendo a sposarsi in nessun altro modo e non riuscendo a denunciare Don Rodrigo, sono riusciti a [...]

La scoperta è stata fatta dai vicini che, dovendosi incontrare colla donna, hanno suonato ripetutamente il campanello e, non sentendo una risposta, hanno iniziato a preoccuparsi.

perché Renzo voleva a tutti i costi sposare Lucia e, non potendolo fare in modo "normale", dovevano raggiungere il prete, 
(3^S, 2012) Zeus vedendo che l'uomo non riusciva a vivere in tranquillità, gli donò giustizia

Vedendo che essi avevano successo apriva un'altra bottega

Dopo di che, ripetendo l'esperimento più volte con ulteriori verifiche, le intuizioni vengono $[\ldots]$

La sera Harry, tornando a casa dal lavoro, non vedendo bene la strada a causa della forte pioggia investe il cane

(5^S, 2012) Crescendo, viaggiò per quasi tutta l'Italia

È per merito della famiglia di animo buono che, adottandolo, il giovane si è costruito una vita

$\left(1^{\wedge} S, 2018\right) \quad$ Prima di Facebook le persone tornando da un viaggio raccontano al famigliare la stessa storia

Accettando la richiesta, sarai in grado di vedere $[\ldots]$

(U 2018/19) Analizzando i difetti della leva del Novecento si potrebbe riorganizzare la durata del servizio,

(U 2020/2021) Non ottenendo comunicazioni apertamente ostative allo svolgimento della trasferta, il 3 ottobre coinvolge il Gabinetto Regionale (incompetente in materia sanitaria)

Secondo l'editore, dunque, il rischio maggiore è che i testi elettronici, divenendo un mero assemblaggio di contenuti ipertestuali, cancellino secoli di cultura del libro

l'uomo, causa dei suoi mali, prendendo coscienza del problema dell'antropocentrismo, possa aiutare l'ambiente, proteggendo così la nostra casa.

Confrontando lo sviluppo psico-sociale di bambini cresciuti in contesti familiari sia etero, sia omo-parentali, la conclusione dell'articolo è in linea con le istanze conservatrici.

Non è peregrino pensare che nel consolidamento di questa struttura nell'uso comune medio, anche scritto, abbiano avuto un ruolo significativo gli stessi docenti di italiano: negli elaborati analizzati il gerundio presente usato iconicamente non è mai segnalato come errore, né ritenuto improprio - il che può avere contribuito alla speculare regressione del gerundio composto (si veda il par. successivo). Sebbene le grammatiche confermino la canonizzazione del gerundio composto, soprattutto nelle tavole di declinazione morfologica dei verbi, l'accettabilità del gerundio presente sintatticamente iconico per esprimere l'anteriorità è ormai entrato nella grammatica silenziosa (Patota, 1997) veicolata dall'insegnante, a sua volta esperiente e artefice dei mutamenti del nuovo standard. È facile rilevare l'uso iconico del gerundio presente anche nel teacher talk, come mostrano questo estratto del corpus KIParla: 
// la cosa bisogna studiarla un attimo ma //studiando un minimo di integrali quello che si vede e' che basta dividere tutto // per // una costante //

Nondimeno il medesimo corpus conferma la persistenza del gerundio composto nel parlato dell'insegnante:

il costume // essendo legato alla dimensione spettacolare // della presentazione del se' // non e' // un // semplice // vestito //

the sartorialist essendo molto seguito // influenzano hanno un'influenza enorme sul // sugli stili contemporanei //

non essendo dunque stata la chiesa potente da occupare la italia ne' avendo permesso che un altro la occupi // e' stata cagione //

\subsection{Il gerundio composto}

Nel paragrafo precedente e in quello introduttivo si è detto della progressiva scomparsa dall'uso comune del gerundio composto per esprimere l'anteriorità di un evento. Oltre alle riserve linguistiche del gerundio composto segnalate da Dardano (1994), Solarino (1996) rilevava che il tempo verbale in questione persisteva nelle scritture formali, per lo più in funzione causale e concessiva.

La casistica estrapolata dal nostro corpus conferma la forte regressione - se non proprio l'estinzione prossima - del gerundio composto, sebbene per i testi formali, taluni di impronta saggistica, sarebbero appunto adeguati. Vi sono pochi affioramenti nelle scritture scolastiche, concentrati - forse non a caso - negli anni posteriori al ripasso grammaticale, solitamente svolto nel biennio: se ne scovano tre negli elaborati di seconda superiore (uno del 2011, due del 2012), tre negli elaborati di terza superiori (2012) e sei negli elaborati di quinta superiore (2012). Sono invece assenti negli elaborati di prima superiore, sia del 2012 sia del 2018, e sono discretamente attestati negli elaborati universitari (vi si danno sei occorrenze, tre delle quali in un unico elaborato).

La scarsa documentazione del gerundio composto trova conferma nei dati estrapolabili dal corpus VINCA, dove affiorano soltanto cinque gerundi composti sui settecentoventinove testi raccolti; di questi soltanto uno occorre in uno scrivente di scuola superiore. Il gerundio composto sembra quindi resistere nelle scritture giovanili finché supportato dalla riflessione metalinguistica strutturata che può offrire la scuola; al di fuori dell'ambiente scolastico gli scriventi, per quanto coinvolti in attività di scrittura vincolata e formale, lo tralasciano.

Il gerundio composto si conferma essere una rarità anche nel corpus VALICO, dove è stato possibile rintracciare solo tre occorrenze distribuite in due testi dall'interlingue intermedio-avanzate.

Quanto alla tipologia semantica, nel nostro corpus predominano i tipi causali (dodici); le occorrenze del tipo concessivo sono esigue (due). Di seguito si riportano i campioni: 
(C) Italiano LinguaDue 2. 2021. M. Dota, L'uso delle subordinate gerundiali nella scrittura scolastica e universitaria

GERUNDIO COMPOSTO CON VALORE CAUSALE

$\left(2^{\wedge} S, 2011\right) \quad$ Essendo stato affidato a una famiglia dalla nascita, i suoi genitori $[\cdots]$

$\left(2^{\wedge} S, 2012\right) \quad[\ldots]$ oppure essendo stati traditi hanno ucciso per vendetta.

Essendo l'epicentro situato probabilmente nelle acque $[\ldots]$ ben presto ai terremoti si aggiungono $[\ldots]$

$\left(3^{\wedge} \mathrm{S}, 2012\right) \quad$ Gli dei avendo trovato l'inganno puniscono Prometeo

e avendo capito che l'uomo avrebbe rischiato l'estinzione, rubò da Atena e Efesto il fuoco

$\left(5^{\wedge} S, 2012\right) \quad$ In quanto lui non avendo trovato né una casa né $[\ldots]$ non può dire cos'era prima

, e avendo girato il mondo successivamente, è spinto a [...]

avendo girato in lungo e in largo si rende conto che [...]

Non avendo vissuto in quell'epoca, non posso $[\ldots]$

[...] in quanto non avendo seguito gli avvertimenti da parte di Nanni di lasciarlo stare lui persevera nel rapporto.

Anguilla, essendosi stanziato presso Gaminella, ha avuto [...]

(U 2018-2019) [...] gli animali e i bambini, che non essendo dotati di ragione, scimmiottano i comportamenti altrui

(U 2020-2021) Avendo quindi analizzato tutto il processo che va dal manifestarsi del disturbo alla guarigione, dovrebbe essere chiaro il pericolo che si corre nel minimizzare questi problemi:

Avendo lavorato vari mesi in una libreria, posso affermare che l'approccio verso il libro è sempre più scontato e distante

varie organizzazioni, come la Starlight Children's Foundation, avendo dovuto sospendere attività di intrattenimento per rispettare le norme anti-Covid, hanno fatto ricorso a postazioni da gaming, [...] nell'articolo di Sirtori si parla di una coppia che, avendo dovuto rimandare il proprio matrimonio a causa della pandemia, ha deciso di organizzarne uno sulla propria isola condivisa [...] Sony, il cui fatturato deriva per il 65\% dalla sola PlayStation, ha dovuto ripensare il day one della nuova PS5, avendo dovuto bloccare le vendite fisiche della console per evitare assembramenti

\section{GERUNDIO COMPOSTO CON VALORE CONCESSIVO}

(2^S, 2012) Pur essendosi dimesso non cancellerà il suo errore.

$\left(3^{\wedge} S, 2012\right) \quad$ esse pur essendo state affrontate in epoche precedenti sono questioni molto attuali 
Si può dunque affermare che il gerundio composto sopravvive in forma relittuale precipuamente nelle scritture scolastiche ed eminentemente nella sua sfumatura causale.

\subsection{Gerundio in proposizioni indipendenti}

Negli elaborati di alcuni studenti di quinta superiore e soprattutto universitari, per quanto votati all'adozione di un registro formale e sorvegliato, si distingue l'impiego del gerundio in proposizioni indipendenti (talvolta reggenti nel periodo che viene a crearsi); il fenomeno viola la natura e la funzione standard intrinseche di questo modo verbale (cfr. par. 2):

(U 2020-2021)

Tutelando così questa completa alimentazione ricca di cereali, frutta, verdura, carne, pesce, latticini e spezie in quantità proporzionate fra loro.

Essendo che la pubblicità è sempre stata lo specchio della società, è giusto affermare che pubblicità e cultura procedono in sintonia, perché nel corso degli anni ha assorbito le tendenze e le dinamiche di ogni paese, di ogni territorio e di ogni epoca. Adeguandosi ai mezzi di comunicazione esistenti, dalla stampa alla radio, dalla televisione al web.

Il fine comunicativo è quello di presentare una tesi personale su tale argomento. Rendendo conscio il lettore di come le dinamiche della comunicazione possano confondere o rappresentare in maniera errata quali sono gli obbiettivi degli Stati Uniti. Invogliando ad un maggiore approfondimento dopo avere letto questo testo. [...] Essi hanno sviluppato nel corso dei decenni una poderosa capacità retorica di mostrarsi agli occhi del mondo come la potenza egemone degli ultimi 100 anni. Cercando però di nascondere il fatto che la loro incontrastata potenza li porti ad essere un impero a tutti gli effetti.

(U 2020/2021) Il vero pero pericolo è quello di vivere in una bolla fatta di nostre convinzioni assecondate da notizie sempre vicine ai nostri ideali e ricerche che mostrano solo informazione a supporto della nostra posizione. Rendendo il web un mondo chiuso, che ci rinchiude sotto una campana di vetro allontanandoci da ogni posizione di scontro o confronto facendoci vivere in un'isola felice che non rappresenta però la realtà.

La diffusione della Biblioteca, determinata da un processo di mercato che riuscì a coprire i diversi interessi dei lettori, è stata testimoniata dai ritrovamenti di questi libri sia nelle campagne che nelle biblioteche private della nobiltà. Permettendo, così, lo sviluppo della cultura popolare dal punto di vista editoriale e creando un nuovo pubblico che comprendeva persone di ogni status sociale.

La piattaforma è libera a tutti e permette a chiunque di condividere i propri contenuti. Venendo retribuiti. Perché si, ad oggi [...] 
La circoscrizione del fenomeno agli studenti più maturi può imputarsi a molteplici fattori: la maggiore sicurezza nella scrittura, vera o presunta, acquisita col completamento del ciclo obbligatorio di istruzione, incoraggia l'affermarsi di uno stile personale, a tratti marcato nella sperimentalità di alcune soluzioni e probabilmente influenzato da una maggiore esposizione - verosimilmente più cospicua rispetto agli anni della piena adolescenza - alla scrittura giornalistica coeva (soprattutto digitale e social) e al suo prototipico stile spezzato (Bonomi, 2016: 209-212; Dota, 2020), quasi sempre esasperato nei lanci d'agenzia. Di seguito un campione esemplificativo tratto da una pagina on line:

Anche se non è stata provata alcuna associazione tra i due eventi, in molti è prevalso evidentemente il senso di paura e smarrimento. Arrivando così a rinunciare ${ }^{8}$.

Certamente vi concorre l'ormai annosa avanzata dello stile nominale (Dardano, 1994 e 2010/2017), che tra le sue risorse contempla l'impiego dei modi non finiti. Lo stilema occorre infatti nell'opera ibrida, tra romanzo e reportage giornalistico, Gomorra di Roberto Saviano: «I clan Casalesi guadagnavano in ogni passaggio dell'economia dell'edilizia. Fornendo cemento, fornendo ditte in subappalto per la costruzione e ricevendo una tangente sui grossi affari» (Roberto Saviano, Gomorra, cit. in Dardano, 2010/2017: 107; corsivi nel testo).

Proposizioni indipendenti col gerundio sono del resto registrate tra le tendenze recenti dell'italiano contemporaneo: il gerundio indipendente avrebbe «molto successo in titoli (Ballando con le stelle)» (D'Achille, 2010); affiora persino nella scrittura espositiva, generalmente sorvegliata, di Wikipedia (Prada, 2016: 106-110), soprattutto in pagine relative a contenuti di cultura popolare, come i manga:

\section{Durante il trasporto avviene uno scambio tra Miaka e Yui, che finisce a Kutou. Scoprendo di essere la sacerdotessa di "Seiryū no miko" ("Sacerdotessa del drago"). (https://it.wikipedia.org/wiki/Fushigi_y\%C3\%Bbgi)}

Come si può inferire anche dagli esempi precedenti, conferire autonomia sintattica a una proposizione progettata col gerundio assegna maggiore salienza, e dunque importanza (se non proprio centralità), a un'informazione che, essendo morfologicamente espressa con un modo non finito - dunque ontologicamente subordinato nel sistema standard -, apparirebbe altrimenti secondaria. In questa tendenza si può leggere tanto una incapacità di gerarchizzare le informazioni, conferendo loro lo statuto sintattico e pragmatico-informativo desiderato per mezzo delle risorse sintattico-testuali standard, quanto, per converso, la pressione dei tratti neostandard e della sua esasperata ricerca dell'espressività a scapito delle soluzioni tradizionali nei (residuali) domini comunicativi non specialistici che pure dovrebbero attenersi a un registro controllato. Il finora mancato intervento correttorio da parte della comunità di contributori al Wiki ${ }^{9}$ conferma infatti l'ampia accettabilità di cui ormai gode questa struttura nell'uso.

${ }^{8}$ Fonte consultabile al link:

https:/ / www.tecnicadellascuola.it/vaccino-astrazeneca-tanti-docenti-non-si-fidano-e-rinunciano-solo-a-treviso -3mila-ci-ripensano-dopo-avere-prenotato.

9 La pagina è stata aperta nel 2006; chi scrive ha consultato la pagina per l'ultima volta il 18/05/2021 e l'intervento più recente risale all'aprile 2021. 


\subsection{L'uso del gerundio subordinativo e i relativi errori in prospettiva acquisizionale}

Gli errori nell'uso del gerundio subordinativo riscontrati nel corpus sono accomunati dallo «sbilanciamento/attrazione verso il nucleo tematico più vicino» (Gualdo, 2010: 42), dalla rievocazione dello «sfondo pragmatico e contestuale» che «nel passaggio dall'oralità alla scrittura viene virtualmente rievocato senza però tener conto della necessità di colmare la sua assenza attraverso adeguati strumenti testuali» (ivi: 46). Di seguito si riproducono i campioni più significativi:

$\left(2^{\wedge} \mathrm{S}, 2011\right) \quad$ Questa scelta, azzardata, lo porta a diventare una delle persone più importanti e innovative di oggi, inventando la Machintosh

basta cambiare leggermente la frase dicendo: “se un' uomo distrugge l'ambiente si chiama costruzione, se l'ambiente distrugge l'uomo si chiama catastrofe" collegandosi alla distruzione delle foreste e al famoso buco nell'ozono

$\left(1^{\wedge} S, 2012\right) \quad$ Luna ci ha fatto presentare davanti alla classe, raccontando qualcosa che ci rappresenta [è lo studente a raccontarsi]

$\left(2^{\wedge} \mathrm{S}, 2012\right) \quad$ l'unica cosa che si può dire è che una donna matura prima di un uomo, questa cosa è dovuta soprattutto ad un diverso modo di pensare e interagire, riuscendo a capire i propri sbagli

Tra i giovani oltre all'abuso di droghe ed alcool si è sviluppata anche la "moda" di iniziare a far sesso fin da giovani non aspettando neanche i diciotto anni facendo sviluppare così, anche tra i giovani, malattie venerie come l'HIV e l'AIDS

(3^S, 2012) Siamo carichi fino alla testa delle stupidaggini che $[\ldots]$ e sembra che tutto questo non ci interessi, facendoci trasportare alla deriva

Inizialmente la ragazza è presa dalla situazione accettando una rosa, ma [...] [il fatto di accettare la rosa è interpretato come prova di coinvolgimento emotivo]

Ma soprattutto la musica è l'accompagnamento di ogni singolo nostro giorno, essa ti entra dentro, non potendone fare più a meno,

I mezzi di comunicazione e anche la scuola dovrebbero riavvicinare $\mathrm{i}$ giovani alla poesia, riscoprendo la passione per testi in cui ognuno può immedesimarsi

$\left(5^{\wedge} \mathrm{S}, 2012\right) \quad$ spiega come S. Francesco sia riuscito a coinvolgere altri uomini come Bernardo d'Assisi, Egidio e Silvestro, anche loro di Assisi, portandoli a vivere anche loro una vita semplice, camminando scalzi e sposando anche la povertà

la giustizia, il diritto e la legalità sono alla base della convivenza civile non possono essere soggette a compromessi, in quanto comporta la perdita della libertà portando maggiori benefici goduti da altri. 
(1^S, 2018) Generalmente i profili su Facebook sono rappresentazioni, non di come una persona è nella realtà, ma di come vorrebbe essere, comunicando agli altri il proprio sé ideale.

L'identità che viene mostrata è la descrizione del nostro ego ideale rischiando a volte di impersonificarsi in ciò che non si è d'avvero

Mentre, con Facebook, la comunicazione non è mirata, finendo così per raccontare la storia a chiunque sia interessato

Viene utilizzato il termine "amici", rappresentando una cerchia di persone con cui ci sia un legame ma senza particolare affinità

Senz'altro si può interpretare la presenza di questi errori come un ulteriore segnale della pressione della modalità orale su quella scritta (Gualdo, 2010: 42), che può talvolta ingannare i medesimi docenti. Questi rilassamenti sintattici, infatti (lo si è già ricordato), sono riscontrabili anche tra persone adulte e istruite. In particolare, i gerundi irrelati o ambigui presenti nelle scritture universitarie possono muovere «da lanci d'agenzia, quasi sempre caratterizzati da una forte nominalizzazione» (Gualdo, 2010: 41). Benché in misura minore, tali errori affiorano pure nel nostro sotto-corpus universitario:

(U 2018/19) Il Prof. Umberto Galimberti ha sottolineato il difetto forse più grave di tutta l'operazione, quello di una sconfitta morale e pedagogica della scuola italiana, sottomessa a logiche materiali quali quelle del lavoro, dimenticando i doveri della formazione dell'individuo

(U2018/19) giovanissimi autori in grado di stravolgere il mezzo nelle sue finalità espressive, garantendogli perciò una nuova linfa e dimostrando [il sogg. è il mezzo] dunque di potersi sempre reinventare.

(U 2020/21) E anche con la successiva riapertura, la popolazione ha iniziato ad avere sempre più timore di entrare in un qualsiasi negozio, comprese le librerie, risultando così in un incremento nell'acquisto dei libri digitali. 
(U 2020/21) Il ruolo dell'archeologo, dunque, è associato alla ricerca e comprensione del passato, contribuendo perciò alla creazione del valore immateriale e materiale del patrimonio culturale storico,

(U 2020/21) Il fattore socioeconomico si posiziona tra gli scalini più alti dei nostri interessi, assistendo così a una produzione volta a un esponenziale aumento della quantità a fronte della diminuzione dei tempi, dei costi e della qualità

(U 2020/21) durante gli ultimi mesi molta gente ha creduto nelle notizie false più disparate, portando anche all'abbandono di cani e gatti

(U 2020/21) tuttavia a causa dello scioglimento dei ghiacci, l'ambiente in continuo cambiamento non permette ai cacciatori di studiare la preda, fallendo la caccia.

(U 2020/21) Alcuni hanno addirittura teorizzato che i primi antenati dell'uomo erano effettivamente vegetariani, questo perché non erano in grado di cacciare l'animale a mani nude fino all'invenzione delle "armi" e cuocerlo, considerando la carne cruda pericolosa, fino all'invenzione del fuoco.

Come rilevato da Gualdo (2010: 41), in alcuni casi «gli antecedenti cui il gerundio si aggancia sono nomi argomentali che indicano un atto di parola (replica, risposta) o un'azione (esordio)»:

(U 2020/21) Si leggono frequentemente sui social network commenti secondo cui il calcio femminile non raggiungerà mai il livello di quello maschile, dimenticando che il secondo ha avuto diversi decenni in più del primo per arrivare dove è oggi

Affiorano inoltre errori conseguenti ad altre contravvenzioni, come la mancanza dell'esplicitazione del soggetto nei gerundi assoluti, che provoca l'ambiguità dell'espressione:

$\left(1^{\wedge} \mathrm{S}, 2012\right) \quad$ Ero in coppia con Sebastiano e quindi l'esercizio è risultato e facile e divertente essendo un ragazzo simpatico.

$\left(3^{\wedge} \mathrm{S}, 2012\right) \quad$ Essi però studiano una dottrina basata sul pensiero ed essendo proprio degli esseri razionali spesso cadono in errore

Oppure lo slegamento tra gerundiale e frase di rango principale può imputarsi alla presenza di altri impliciti che, se verbalizzati (come si fa di seguito, in corsivo), costituirebbero il tassello di congiunzione tra le due proposizioni: «Basandosi sugli studi scientifici di R. Zhao [si può affermare che] i profili di Facebook non sono altro che la rappresentazione $[\ldots] \gg$.

Nel corpus figurano inoltre gerundi paraipotattici, soprattutto nelle classi inferiori, quale segnale inequivocabile di incertezze nella subordinazione: 
$\left(2^{\wedge}\right.$ S, 2012) Bisognerebbe avere la parità dei sessi senza discriminazione e cercando di abolire questo gravissimo fenomeno

suona la campana, segno che il curato era in pericolo e costringendo, con questo gesto, Renzo e Lucia alla fuga.

Lui dice a Renzo di non fare quelle meditazioni perché Dio avrebbe punito anche lui e dicendo che se ne occupava lui

$\left(1^{\wedge} S, 2018\right) \quad$ pare che Facebook renda maggiormente ardua la creazione di una propria presentazione veritiera, siccome può venirsi a creare un profilo unico rivolto a tutti gli utenti che non rispecchi la selettività di racconto del soggetto al fine di fare emergere la caratteristica sulla quale si vuole focalizzare l'attenzione dello spettatore ed estraniando il rapporto "faccia a faccia",

(U 2020/21) in più, si teme di perdere il pubblico in presenza, dato il ragionamento: "Ho già visto lo spettacolo in tv, non serve vederlo dal vivo", ed essendo meno costoso rivedere il filmato rispetto al prezzo elevato dei biglietti.

La scarsa confidenza con l'ipotassi, col concorso dell'uso iconico del gerundio presente e di svarioni lessicali, possono poi produrre periodi apparentemente corretti sul piano morfosintattico, ma infelici sul piano comunicativo e semantico:

( $\left.3^{\wedge} S, 2012\right) \quad$ Bruce Wayne, rimane orfano da piccolo, vedendo la morte dei suoi genitori in faccia, decide così di affrontare la criminalità, cercando di debellarla o aiutandola ad uscire da quella vita.

Adottando una prospettiva acquisizionale, può essere proficuo domandarsi a quale livello del QCER per l'italiano L2/LS si considerano padroneggiabili le subordinate al gerundio. Anna Giacalone Ramat (2003) ha dimostrato che il gerundio compare piuttosto tardi nelle produzioni degli apprendenti che, nel caso delle subordinate, preferiscono a lungo le equivalenti esplicite. I diversi usi del gerundio comparirebbero, infatti, secondo la sequenza acquisizionale gerundio nella perifrasi progressiva $>$ gerundio di predicato $>$ gerundio di frase.

Se consideriamo le principali certificazioni di italiano, il gerundio compare per la prima volta nella combinazione perifrastica con stare per il livello B1, conseguito il quale l'apprendente dovrebbe essere in grado di usare correttamente alcune frasi subordinate in forma principalmente esplicita, ad eccezione delle finali, per le quali è prevista anche la padronanza della forma implicita. Per le medesime certificazioni il gerundio presente e passato nelle frasi implicite è proposto per il conseguimento del livello C1; ne consegue che per approcciare l'argomento con profitto, l'apprendente dovrebbe già pienamente attestarsi sul livello intermedio più avanzato (B2).

Analoga è la collocazione dell'argomento "gerundio subordinato" nella manualistica di italiano L2 (cfr. Duso, 2021: par.7): a titolo esemplificativo, citiamo il manuale Loescher Viaggio nell'italiano. Corso di lingua e cultura italiana per stranieri (2004), nel quale gli usi subordinati del gerundio sono collocati nella penultima unità (su dodici totali) per il conseguimento del livello $\mathrm{C} 1$; in Magari. Corso di lingua e cultura italiana di livello intermedio e avanzato (2008) il gerundio è situato nella prima nella prima unità di livello C1, insieme agli 
altri modi indefiniti; anche nel fortunato Espresso (2005) la trattazione degli usi subordinati del gerundio è diluita nei dieci capitoli che costituiscono il volume di livello avanzato.

Poiché possiamo considerare l'italiano in formazione degli adolescenti nativi di italiano una interlingua, per la nostra riflessione potrebbe essere produttivo adottare, anche per l'italiano L1, l'approccio acquisizionale consolidato per l'italiano L2. Del resto, se considerassimo quali sono le abilità di produzione attese al termine della scuola secondaria inferiore, vedremmo che esse sono pressocché parificabili a quelle previste per il livello B1 del QCER; per quanto i traguardi e gli obiettivi previsti dalle Indicazioni nazionali (2012) per l'italiano L1 della classe terza media non siano articolati in descrittori puntuali come per l'italiano L2, questi ultimi, relativi al livello B1, sembrano calzare ugualmente: per la produzione orale il diplomato di terza media sostanzialmente «sa descrivere, collegando semplici espressioni, esperienze e avvenimenti, le sue aspirazioni, le sue speranze e le sue ambizioni. Sa motivare e spiegare brevemente opinioni e intenzioni. Sa narrare una storia breve, la trama di un libro o di un film, indicandone i punti salienti e descrivere le sue impressioni»; per la produzione scritta «Sa scrivere semplici testi coerenti su argomenti noti e di suo interesse. Sa scrivere lettere personali esponendo esperienze e impressioni».

A biennio superiore concluso, al netto della difformità di obiettivi previsti dalle Indicazioni nazionali per i diversi indirizzi (per un sunto cfr. Dota, Lugarini, 2018: 644-653), le competenze linguistiche maturate nell'uso dell'italiano sono idealmente e grosso modo accostabili alle abilità padroneggiate al livello B2 del QCER, che si articolano come riepilogato di seguito (si vedano, in particolare, le attività di produzione):

Tabella 2. Descrizione generale delle competenze per il livello B2 (adattato da QCER: 44-45)

\begin{tabular}{|c|c|c|c|c|c|}
\hline & \multicolumn{2}{|c|}{ COMPRENSIONE } & \multicolumn{2}{|c|}{ PARLATO } & \multirow{2}{*}{$\begin{array}{c}\text { SCRITTO } \\
\begin{array}{c}\text { Produzione } \\
\text { scritta }\end{array} \\
\end{array}$} \\
\hline & Ascolto & Lettura & Interazione & Produzione orale & \\
\hline B2 & $\begin{array}{l}\text { Comprende } \\
\text { discorsi di una } \\
\text { certa estensione e } \\
\text { conferenze. È in } \\
\text { grado di seguire } \\
\text { argomentazioni } \\
\text { anche complesse } \\
\text { purché } \\
\text { l'argomento gli sia } \\
\text { relativamente } \\
\text { familiare. Capisce } \\
\text { la maggior parte } \\
\text { dei notiziari e } \\
\text { delle trasmissioni } \\
\text { TV che riguardano } \\
\text { fatti di attualità. } \\
\text { Riesce a capire la } \\
\text { maggior parte dei } \\
\text { film in lingua } \\
\text { standard. }\end{array}$ & $\begin{array}{l}\text { Comprende } \\
\text { articoli, servizi } \\
\text { giornalistici, } \\
\text { relazioni su } \\
\text { questioni di } \\
\text { attualità in cui } \\
\text { l'autore prende } \\
\text { posizione ed } \\
\text { esprime un punto } \\
\text { di vista } \\
\text { determinato. } \\
\text { Riesce a } \\
\text { comprendere un } \\
\text { testo narrativo } \\
\text { contemporaneo } \\
\text { anche se di una } \\
\text { certa lunghezza. }\end{array}$ & $\begin{array}{l}\text { Riesce a } \\
\text { comunicare con un } \\
\text { grado di } \\
\text { spontaneità e } \\
\text { scioltezza } \\
\text { sufficiente per } \\
\text { interagire in modo } \\
\text { spontaneo con } \\
\text { parlanti nativi. } \\
\text { Riesce a } \\
\text { partecipare } \\
\text { attivamente a una } \\
\text { discussione in } \\
\text { contesti familiari, } \\
\text { esponendo e } \\
\text { sostenendo le sue } \\
\text { opinioni. }\end{array}$ & $\begin{array}{l}\text { Riesce ad } \\
\text { esprimersi in } \\
\text { modo chiaro e } \\
\text { articolato su una } \\
\text { vasta gamma di } \\
\text { argomenti che lo } \\
\text { interessano. } \\
\text { Sa esprimere } \\
\text { un'opinione su un } \\
\text { argomento } \\
\text { d'attualità, } \\
\text { indicando } \\
\text { vantaggi e } \\
\text { svantaggi delle } \\
\text { diverse opzioni. }\end{array}$ & $\begin{array}{l}\text { È in grado di } \\
\text { scrivere testi chiari } \\
\text { e articolati su } \\
\text { un'ampia gamma } \\
\text { di argomenti che } \\
\text { gli interessano. } \\
\text { É capace di } \\
\text { scrivere brevi } \\
\text { relazioni, fornendo } \\
\text { informazioni e } \\
\text { ragioni a favore o } \\
\text { contro una } \\
\text { determinata } \\
\text { opinione. Sa } \\
\text { scrivere lettere } \\
\text { mettendo in } \\
\text { evidenza il } \\
\text { significato che } \\
\text { attribuisce } \\
\text { personalmente agli } \\
\text { avvenimenti e alle } \\
\text { esperienze. }\end{array}$ \\
\hline
\end{tabular}

Sebbene gli studenti considerati nel corpus siano quasi esclusivamente italofoni nativi, l'insorgere e il successivo fossilizzarsi di questi errori, in questa prospettiva, rivendicano almeno la possibilità di una chiave di lettura alternativa: tra gli studenti del biennio superiore, per questa struttura, in relazione alla padronanza linguistica verosimilmente da 
loro raggiunta, prevale ancora la più immediata modalità di strutturazione testuale semantico-pragmatica poiché, a dispetto delle ore di teoria grammaticale accumulate negli anni, all'atto della produzione lo studente non è sempre in grado di attivare correttamente la modalità sintattica. L'assenza di una riflessione esplicita su questi aspetti può poi provocarne la fossilizzazione e il perdurare nell'babitus scrittorio dei più adulti, al contempo sedotti dai più attrattivi usi mediali, «assordanti» rispetto alla «reticenza normativa» delle grammatiche scolastiche e della didattica (Gualdo, 2010: 46).

Nella manualistica di italiano L2, al contrario, le subordinate implicite al gerundio sono presentate attraverso un approccio didattico induttivo, più consolidato nella manualistica di italiano L2 rispetto alla gemella in L1; nel già citato manuale Loescher per i livelli B2/C1, ad esempio, a partire da un testo di natura saggistica (quindi informativo-argomentativo), lo studente deve individuare le forme verbali coniugate al gerundio, ricavando dal cotesto il loro valore semantico e funzionale, per poi verificare la loro sostituibilità con proposizioni esplicite equivalenti. L'osservazione guidata, inoltre, permette allo studente di ricavare e quindi riflettere sull'obbligatorietà della coreferenzialità del soggetto e sulla posposizione degli eventuali soggetti pronominali nei costrutti assoluti.

L'antidoto al cronicizzarsi del gerundium pendens nell'uso medio potrebbe allora consistere nell'adozione di un approccio modellato sulle grammatiche pedagogiche (Duso, 2007: 137-154; Lugarini, Dota, 2018: 550-554) anche per l'italiano L1: nell'ora di grammatica non bisognerebbe limitarsi a presentare la morfologia del gerundio e le subordinate gerundiali nella carrellata indifferenziata della tassonomia morfologica o delle proposizioni, bensì osservare il gerundio subordinativo in contesto, in relazione alle consuetudini e possibilità d'uso nei diversi generi testuali; in luogo di incoraggiare all'ambiguità indistinta per aggirare le normali difficoltà di strutturazione sintattica, nelle attività di produzione scritta analogamente alla didattica in italiano L2 - si dovrebbe far consolidare dapprima la subordinazione esplicita, per poi considerare le subordinate equivalenti al gerundio, la loro maggiore o minore adeguatezza a certi stili, scopi e tipologie testuali. Tale approccio gioverebbe inoltre all'osservazione e al consolidamento di quelle sfumature semantiche del gerundio oggi più peregrine nella scrittura scolastica.

\section{RIFERIMENTI BIBLIOGRAFICI}

Antonelli G. (2011), "Lingua", in Afribo A., Zinato E. (a cura di), Modernità italiana. Cultura, lingua e letteratura dagli anni settanta a oggi, Carocci, Roma, pp. 15-52.

Antonini A. (1974), "Il problema del gerundio", in Studi di grammatica italiana, IV, pp. 85-107. Barattella M. (2015-2016), Ragionando sul gerundio con i bambini della scuola primaria, tesi di laurea (relatrice: Maria Giuseppa Lo Duca), Università degli Studi di Padova.

Bertinetto P. M. (1986), Tempo, aspetto e azione nel verbo italiano. Il sistema dell'indicativo, Accademia della Crusca, Firenze.

Bonomi I. (2016), "La lingua dei quotidiani”, in Bonomi I., Morgana S. (a cura di), La lingua italiana e i mass media, Carocci, Roma, pp. 167-220. 
Caddeo R. (2019), “Aspetti dell'interferenza sardo-italiano: il gerundio nell'italiano regionale di Sardegna", in Rhesis. International journal of Linguistic, Philology and Literature, 10, 1, pp. 75-112.

Cortelazzo M. (2002), "Gerundio a doppio taglio", in Guida agli Enti Locali, p. 88.

Cortelazzo M. (2014a), "Gerundio", in Parole. Opinioni, riflessioni, dati sulla lingua: https://cortmic.myblog.it/gerundio/.

Cortelazzo M. (2014b), "L'italiano nella scrittura amministrativa", in Lubello S. (a cura di), Lezioni d'italiano. Riflessioni sulla lingua del nuovo millennio, il Mulino, Bologna, pp. 85-104.

Cortelazzo M. (2015a), "La semplificazione dei testi amministrativi. Le buone pratiche", in Bombi R. (a cura di), Quale comunicazione tra stato e cittadino oggi? Per un nuovo manuale di comunicazione istituzionale e internazionale, Il calamo, Roma, pp. 93-110.

Cortelazzo M. (2015b), "Il cantiere del linguaggio istituzionale. A che punto siamo?", in LeGes XXVI (2015), pp. 135-150.

D'Achille P. (2010), “Lingua d'oggi”, in Enciclopedia dell'italiano online: http://www.treccani.it/enciclopedia/lingua-d-oggi_(Enciclopedia-dell’Italiano)/.

Dardano M. (1994), "Profilo dell'italiano contemporaneo", in Serianni L., Trifone P. (a cura di), Storia della lingua italiana, vol. II, Einaudi, Torino, pp. 343-430.

Dardano M. (2012), (a cura di), Sintassi dell'italiano antico: la prosa del Duecento e del Trecento, Carocci, Roma.

Dardano M. (2010/2017), Stili provvisori: la lingua nella narrativa italiana d'oggi (2005-09), Carocci, Roma.

Dardano M. (2020), (a cura di), Sintassi dellitaliano antico 2: la prosa del Duecento e del Trecento: la frase semplice, Carocci, Roma.

De Roberto E. (2013), "Usi concorrenziali di infinito e gerundio in italiano antico", in Casanova Herrero E., Calvo Rigual C. (a cura di), Atti del XXVI congresso di linguistica e filologia romanza, De Gruyter, Berlin-New York, II, pp. 878-888.

Dota M. (2020), "Lo storytelling giornalistico su Instagram: un'analisi testuale", in Piotti M., Prada M. (a cura di), A carte per aria: Problemi e metodi dell'analisi linguistica dei media, Franco Cesati, Firenze, pp. 233-243.

Dota M., Prada M. (2020), "Un'esperienza di didattica "verticale" della scrittura: il PON Monticello", in Italiano LinguaDue 12 (2020), 1, pp. 303-340:

https:/ / riviste.unimi.it/index.php/promoitals/article/view/14019.

Duso E. M. (2007), Dalla teoria alla pratica: la grammatica nella classe di italiano L2, Aracne, Roma.

Duso E. M. (2021), "Il gerundio nell'apprendimento e insegnamento dell'italiano L2 a studenti in scambio e internazionali", in Italiano LinguaDue 13 (2021), 1, pp. 282-318.

Dressler W., Mayerthaler W., Panagl O., Wurzel W. (1987), Leitmotifs in Natural Morphology, Amsterdam, Benjamins.

Egerland V. (2010a), "Gerundio", in Enciclopedia dellitaliano (ENCIT), Istituto della Enciclopedia italiana, Roma, pp. 570-73:

https://www.treccani.it/enciclopedia/gerundio_(Enciclopedia-dell'Italiano)/.

Egerland V. (2010b), "Frasi subordinate al gerundio", in Salvi, Renzi (2010), pp. 903-920.

Fornaciari R. (1884), Sintassi italiana dell'uso moderno, G. C. Sansoni, Firenze.

Frenguelli G. (2003), "Tra narrazione e argomentazione: il gerundio nella prosa d'arte dei primi secoli", in Il verbo italiano. Studi diacronici, sincronici, contrastivi, didattici. Atti del 35. 
(C) Italiano LinguaDue 2. 2021. M. Dota, L'uso delle subordinate gerundiali nella scrittura scolastica e universitaria

Congresso internazionale di studi [Della SLI, Società di linguistica italiana]. Parigi, 20-22 settembre 2001, Bulzoni, Roma, pp. 23-42.

Giacalone Ramat A. (2003), "Gerunds and optional categories in second language learning", in Ead. (ed.), Typology and second Language Acquisition, Mouton de Gruytier, Berlin-New York, pp. 181-220.

Goidànich P.G. (1919), Grammatica italiana ad uso delle scuole: con nozioni di metrica esercizi e suggerimenti didattici, Zanichelli, Bologna.

Gualdo R. (2010), Per l'italiano: saggi di storia della lingua nel nuovo millennio, Aracne, Roma.

Lonzi L. (1991), "Frasi subordinate al gerundio", in L. Renzi, G. Salvi, A. Cardinaletti (a cura di), Grande grammatica italiana di consultazione, il Mulino, Bologna, 1988-1995, 3 voll., vol. $2^{\circ}$ (I sintagmi verbale, aggettivale, avverbiale; la subordinazione), pp. 571-592.

Lubello S. (2015), "Ancora sull'italiano burocratico. Riflessioni sulla base di un corpus recente", Studi di grammatica italiana, XXXIV (2015), pp. 263-282.

Lubello S. (2018), "L'antilingua gode di buona salute: nuove forme, vecchi vizi", in Sergio G. (a cura di), Comunicare cittadinanza nell'era digitale Saggi sul linguaggio burocratico 2.0, FrancoAngeli, Milano, pp. 31-43.

Lugarini E., Dota M. (2018), Grammatica dell'italiano: fondamenti e metodi per l'insegnamento e l'apprendimento della lingua italiana L1 e L2, EdiSes, Napoli.

Mayerthaler W. (1981), Morphological Naturalness, Karoma, Ann Arbor.

Morandi L., Cappuccini G. (1895), Grammatica italiana per uso delle scuole: volume unico: regole ed esercizi, G. B. Paravia, Torino etc.

Patota G. (1997), "La grammatica silenziosa", in Norma e lingua in Italia: alcune riflessioni fra passato e presente, Istituto lombardo di scienze e lettere, Milano, pp.71-112.

Petrocchi P. (1887), Grammatica della lingua italiana: per le scuole ginnasiali, tecniche, militari ecc., Treves, Milano.

Policarpi G. (1974), “Tipi di proposizione e di periodo nell'italiano popolare contemporaneo e in Croce", in Medici M., Sangregorio A. (a cura di), Fenomeni morfologici e sintattici nell'italiano contemporaneo, Bulzoni, Roma, pp. 651-716.

Prada M. (2015), L'italiano in rete: usi e generi della comunicazione mediata tecnicamente, FrancoAngeli, Milano.

Renzi L., Salvi G., Cardinaletti A. (1988-1995), (a cura di), Grande grammatica italiana di consultazione, il Mulino, Bologna, 3 voll.

Rohlfs G. (1966-69), Grammatica storica della lingua italiana e dei suoi dialetti, Einaudi, Torino, 3 voll.

Rossi F. (2009), "La perifrasi aspettuale stare + gerundio in costrutti subordinati impliciti", in Sintassi storica e sincronica dell'italiano: subordinazione, coordinazione, giustapposizione. Atti del X Congresso della Società internazionale di linguistica e filologia italiana, Basilea, 30 giugno-3 luglio 2008, Franco Cesati, Firenze, pp. 1155-1170.

Ruele M., Zuin E. (2020), Come cambia la scrittura a scuola: rapporto di ricerca, IPRASE, Provincia autonoma di Trento-Rovereto.

Sabatini F. (2009), "Uso del gerundio con soggetto diverso da quello della frase reggente", in La Crusca per voi 38:

https://accademiadellacrusca.it/it/consulenza/uso-del-gerundio-con-soggetto-divers o-da-quello-della-frase-reggente/268.

Salvatore E. (2019), “Imparare a imparare': osservazioni sull'insegnamento universitario della scrittura", in Italiano LinguaDue 12 (2020), 1, pp. 65-89: 
(C) Italiano LinguaDue 2. 2021. M. Dota, L'uso delle subordinate gerundiali nella scrittura scolastica e universitaria

https://riviste.unimi.it/index.php/promoitals/article/view/13982.

Salvi G., Renzi L. (2010), Grammatica dellitaliano antico, il Mulino, Bologna, 2 voll.

Salvi G., Vanelli L. (2004), Nuova grammatica italiana, il Mulino, Bologna.

Schwarze C. (2009), Grammatica della lingua italiana, Edizione italiana interamente riveduta dall'autore a cura di Adriano Colombo, Carocci, Roma (ed. originale 1988, Grammatik der italienischen Sprache, Niemeyer, Tubingen).

Serianni L. (2000), Italiano. Grammatica, sintassi, dubbi, Garzanti, Torino.

Serianni L. (2006/2017), Prima lezione di grammatica, Laterza, Bari.

Serianni L. (2013), Leggere scrivere argomentare [Prove ragionate di scrittura], Laterza, Roma-Bari.

Skytte G. (1991), "Il gerundio nel quadro della grammatica italiana. Un caso critico negli studi di linguistica italiana", in Varvaro A. (a cura di), La linguistica, oggi, Bulzoni, Roma, pp. 177-181.

Solarino R. (1991), "Il gerundio si espande perché è un’icona", in Italiano e Oltre, 5/91, pp. 219-223.

Solarino R. (1992), "Fra iconicità e paraipotassi: il gerundio nell'italiano contemporaneo", in Moretti B., Petrini D., Bianconi S. (a cura di), Linee di tendenza dell'italiano contemporaneo, Bulzoni, Roma, pp. 155-170.

Solarino R. (1996), I tempi possibili. Le dimensioni temporali del gerundio italiano, Unipress, Padova.

Trifone M. (2006/2009), "Il linguaggio burocratico", in Trifone P. (a cura di), Lingua e identità. Una storia sociale dell'italiano, Carocci, Roma, pp. 213-240.

Virdis M. (1983), "Note sul gerundio nelle lingue neolatine", in Annali della facoltà di lettere e filosofia. Nuova serie IV (XLI), pp. 149-173.

Voghera M. (1985), "Alcune considerazioni statistiche e funzionali sulla subordinazione nell'italiano contemporaneo", in Franchi De Bellis A., Savoia L.M. (a cura di), Sintassi e morfologia della lingua italiana d'uso. Teorie e applicazioni descrittive, Bulzoni, Roma, pp. 421-26.

Wurzel W. U. (1989), Inflectional Morphology and Naturalness, AkademieVerlag, Berlin.

\section{Corpora}

KIParla = Mauri C., Ballarè S., Goria E., Cerruti M., Suriano F. (2019), "KIParla corpus: a new resource for spoken Italian”, in Bernardi R., Navigli R., Semeraro G. (eds.), Proceedings of the 6th Italian Conference on Computational Linguistics CLiC-it. http://kiparla.it/.

VALICO = Varietà Apprendimento Lingua Italiana Corpus On line: http://www.valico.org/.

VINCA = Varietà di Italiano di Nativi Corpus Appaiato: http://www.valico.org/vinca.html. 